\title{
Mandenkan
}

MANDENIKAN Bulletin semestriel d'études linguistiques mandé

$50 \mid 2013$

Numéro 50

\section{Les nouveaux sites Internet de la communauté soninké et la standardisation de la langue}

The new Web sites of the Soninke community and their impact on the standardization of the language

НОВЫЕ ИНТЕРНЕТ-САЙТЫ ЯЗЫКОВОГО СООБЩЕСТВА СОНИНКЕ И

СТАНДАРТИЗАЦИЯ ЯЗЫКА

Gérard Galtier

\section{(2) OpenEdition}

Journals

Édition électronique

URL : https://journals.openedition.org/mandenkan/231

DOI : 10.4000/mandenkan.231

ISSN : 2104-371X

Éditeur

Llacan UMR 8135 CNRS/Inalco

Édition imprimée

Date de publication : 1 décembre 2013

Pagination : 39-60

ISSN : 0752-5443

\section{Référence électronique}

Gérard Galtier, « Les nouveaux sites Internet de la communauté soninké et la standardisation de la langue », Mandenkan [En ligne], 50 | 2013, mis en ligne le 01 décembre 2013, consulté le 08 juillet 2021. URL : http://journals.openedition.org/mandenkan/231 ; DOI : https://doi.org/10.4000/ mandenkan.231

Ce document a été généré automatiquement le 8 juillet 2021.

Les contenus de Mandenkan sont mis à disposition selon les termes de la Licence Creative Commons Attribution - Pas d'Utilisation Commerciale - Partage dans les Mêmes Conditions 4.0 International. 


\section{Les nouveaux sites Internet de la communauté soninké et la standardisation de la langue}

The new Web sites of the Soninke community and their impact on the standardization of the language

НОВЫЕ ИНТЕРНЕТ-САЙТЫ ЯЗЫКОВОГО СООБЩЕСТВА СОНИНКЕ И

СТАНДАРТИЗАЦИЯ ЯЗЫКА

Gérard Galtier

\section{NOTE DE L'AUTEUR}

Cet article est basé sur notre communication (de même titre) délivrée lors du troisième colloque international « Langues et linguistique mandé », qui s'est tenu du 14 au 17 septembre 2011 dans les locaux du laboratoire LLACAN du CNRS, à Villejuif. Le présent texte a été remis à jour en septembre 2012.

1 Le développement d'Internet a eu un développement inattendu : c'est de procurer une nouvelle vitalité à des langues en diaspora. Par exemple, on a constaté une grande pénétration sur le Web de langues telles que l'arménien ou le judéo-espagnol (appelé aussi ladino) [Koulayan 2006]. Le phénomène est mondial: c'est ainsi que de nombreuses langues indiennes d'Amérique du Sud ont trouvé elles aussi leur place sur Internet, notamment pour recréer des liens avec les migrants partis à l'étranger (Gomez Mont 2007).

2 Tournons-nous maintenant vers l'Afrique et faisons sur Internet une recherche sur la langue mungaka de la chefferie de Bali, qui avait été utilisée au début du siècle par la mission protestante de Bâle comme langue commune pour l'évangélisation de ce qui est maintenant la province Nord-Ouest du Cameroun (région anglophone dont la capitale est Bamenda). On arrivera alors sur un site basé non pas au Cameroun, mais aux États- 
Unis (balinyonga.com). Ce site a été développé par des immigrés de la chefferie de Bali soucieux de transmettre leur culture ancestrale à leurs enfants.

De même, grâce aux nouveaux médias, la diaspora soninké joue actuellement un grand rôle dans le développement de sa langue et le maintien de son identité.

Dans la première partie de cet article, nous présenterons un panorama des nouveaux médias soninké, puis dans la seconde partie, nous examinerons, par voie de conséquence, le rôle d'Internet dans la standardisation du soninké écrit.

\section{$1^{\text {re }}$ partie. Panorama des nouveaux médias soninké}

5 Les Soninkés sont originaires des régions frontalières du Mali, de la Mauritanie et du Sénégal. Il existe aussi des communautés importantes en Gambie et en Côte d'Ivoire. Or, le fait que les Soninkés ne soient dominants numériquement dans aucun de ces pays et qu'ils constituent une importante diaspora (c'est la principale communauté africaine en France) les incite à renforcer leur identité ethnique au détriment de leur appartenance nationale. De plus, des provinces traditionnelles soninké telles que le Gadyaga et le Guidimakha sont divisées entre le Mali et le Sénégal pour la première, et entre le Mali et la Mauritanie pour la seconde.

Depuis les années 1980, la langue soninké occupe une place importante dans divers médias de type oral. Ainsi, il existe une importante discographie soninké (cassettes et CDrom) vendue dans toute la communauté. Il existe aussi des films en soninké, un peu artisanaux et enregistrés sur DVD, qui connaissent un grand succès. Dans les radios rurales très présentes en Afrique occidentale, le soninké a également trouvé sa place. On peut par exemple citer la "Radio rurale de Kayes " (au Mali), particulièrement active, qui émet essentiellement en soninké.

7 En ce qui concerne le domaine de l'écrit, on ne sera pas étonné que les Soninkés et leur langue aient eux aussi fait leur apparition sur Internet. On constate que les utilisateurs sont dispersés dans le monde entier ; cela permet de maintenir des liens entre tous les membres de la diaspora. En 2012, on dénombre tout à la fois des sites Web consacrés à la langue et à la culture soninké, des groupes Facebook qui se réclament de la langue et de la culture soninké, des sites transnationaux, des sites de municipalités dans le pays soninké, des sites d'ONG travaillant en pays soninké et des blogs personnels (souvent musicaux) se réclamant d'une identité soninké.

8 Notre corpus de départ comprenait une quarantaine de sites et de blogs spécifiquement soninké ou installés dans des régions soninké. Pour le présent article, nous avons retenu uniquement ceux qui se consacrent de façon importante ou partielle à la langue et à la culture soninké (treize sites d'associations et cinq blogs de militants de la langue et de la culture). Ces sites et blogs peuvent être localisés aussi bien en Afrique qu'à l'extérieur du continent.

9 Nous avons ainsi exclu de cette recherche un certain nombre de sites, centrés sur les questions de développement, qui sont entièrement en français et qui ne contiennent que très rarement des textes en soninké ou sur la langue soninké.

10 Nous n'avons pas non plus retenu douze blogs musicaux ou festifs. Il s'agit de blogs communautaires fréquentés par les jeunes Soninkés, qui présentent essentiellement de la musique moderne, des chansons et de nombreuses photos. 


\section{Un regard sur Facebook}

11 L'étude approfondie des groupes soninké créés sur Facebook ne fait pas partie de cet article; néanmoins, il est nécessaire de les mentionner. En effet, il y a un nombre considérable de groupes soninké sur Facebook. Ils évoluent beaucoup, d'autant que la direction de ce «réseau social » supprime périodiquement ceux qu'elle juge trop peu actifs. Fin septembre 2012, nous avons dénombré environ 125 groupes de plus de 10 membres, qui se réclament d'une identité soninké ${ }^{1}$.

12 À cette date, les groupes Facebook les plus importants numériquement (avec plus de 1000 membres) étaient les sept suivants : le groupe «Mouvement Soninkara Wagadou Rèmou " avec 4154 membres; le groupe «Marenmou Soninkho o Yanibaane Yade " avec 3294 membres; le groupe «Soninkara Gnime: La Racine Soninké » avec 2230 membres; le groupe «Sooninkara Reemou (liberté d'expression)» avec 1867 membres ; le groupe « Soninkara.com » avec 1283 membres ; le groupe «Rap Soninké » avec 1252 membres; le groupe "Soninkaxu » avec 1035 membres $^{2}$. Dans ces groupes, tous les sujets sont abordés, et pas uniquement les questions linguistiques.

13 Il existe aussi des groupes plus restreints, qui se consacrent uniquement à la langue, notamment, en fin septembre 2012 : le groupe «Apprendre le Soninké de base » avec 833 membres; le groupe "Académie Soninké" avec 252 membres; le groupe «Soninkara: Proverbes / Citations Soninké » avec 220 membres; le groupe "Sooninke Renme an Xannen Safa " avec 102 membres $^{3}$. Ces groupes, plus sélectifs ou plus spécialisés, regroupent moins de membres.

14 Les langues utilisées dans ces groupes sont soit le français (en majorité), soit le soninké écrit avec une orthographe française, soit le soninké écrit selon les normes officielles (c'est, entre autres, le cas dans les groupes restreints cités ci-dessus).

15 Examinons maintenant les treize sites et les cinq blogs que nous avons retenus pour la présente étude.

\section{Le site Soninkara.com}

[http://www.soninkara.com]

16 Le plus important des treize sites analysés est « Soninkara.com ». Il est basé en France et son principal animateur est Fodyé Cissé, informaticien originaire du Sénégal. Le site fut fondé en 2001 par un groupe d'internautes qui s'étaient rencontrés sur les forums de Seneweb ${ }^{4}$. Mais, en 2006, suite à un conflit interne, une scission s'est produite qui a donné naissance à un second site, « Soninkara.org ».

Les visiteurs du site Soninkara.com sont principalement des jeunes Soninkés nés en France, mais le site est aussi consulté depuis l'Afrique et tous les pays de la diaspora. D'après ce qui est indiqué, il y a plus de 10000 personnes inscrites sur le forum.

À partir de Soninkara.com, il est possible de se connecter à treize chaînes de radios (telles que les radios locales «Jiida» de Dakar et "Jikke» de Waoundé) et à neuf chaînes de télévision. Il s'agit là de chaînes africaines qui ne sont pas spécifiquement soninké.

Le site Soninkara.com porte en sous-titre la mention «Le portail du peuple Soninké (Sénégal, Mali, Mauritanie, Gambie) » : il se présente ainsi comme un site international ouest-africain, bien qu'il soit basé en France. L'axe du site est essentiellement la 
défense et la promotion de la culture soninké, ce qui correspond aux diverses rubriques.

Le site ne perçoit aucune subvention, mais il est très équilibré du point de vue financier grâce à la publicité qu'il reçoit.

Voilà la liste des rubriques et sous-rubriques :

- Histoire : Histoire, Géographie ; Légendes.

-Société : Organisation sociale; Mariage ; Circoncision; Religion; Environnement ; Migrations ; Cuisines et recettes ; Thèses et mémoires.

- Langue: Apprendre le soninké; Émissions en soninké; Écoles de soninké; Articles en soninké ; DPLN ; Glossaire de mots ; La Voix de l'APS.

- Culture : Musique et danse ; Musiciens soninké ; Contes et poèmes ; Sonink-ART ; APS.

- Artisanat : Teintures ; Sculpture ; Outils traditionnels.

-Informations : Actualités; Littérature; Bibliographie; Associations; Hommage ; Événements; Sport ; Soninkara Xibaaru.

- Contact : Nous contacter; Livre d'Or.

-Autres : Forum; CHAT; Téléchargements; Galeries d'images; Liens; Proposer un lien; Ancien forum; Ancien CHAT; Proposer un article; Pétitions; Plan du site; Plan du site Actualités ; TV / Radios ; Centre multimédia ; Radio Jiida FM Dakar.

La langue soninké est très présente sur le site Soninkara.com. On y trouve ainsi de nombreux textes en soninké : dans la rubrique "Langue " bien sûr, et aussi dans les sous-rubriques «Légendes » et "Contes et poèmes ». Dans le forum, les débats se font souvent en soninké.

Il est possible de télécharger plusieurs des textes en soninké qui sont présentés. Dans la rubrique "Littérature ", on trouve l'enregistrement d'une interview de Yaya Camara, " soldat de la langue et la culture soninké ». Est aussi présent un émule de Cheikh Anta Diop, Adama Dramé, qui explique les liens entre l'égyptien ancien et le soninké. Dans le forum, il y a de nombreux débats sur la langue soninké : par exemple, sur le thème "Pourquoi les jeunes de France mettent-ils le [ra] à la place du [xa] en soninké ». On trouve également une copie $d u$ rapport $d u$ "Séminaire sous-régional sur l'harmonisation de l'orthographe du soninké », tenu à Bakel en novembre 1995.

Les intervenants sont libres d'écrire le soninké, sans contrainte orthographique particulière. Dans l'ensemble, les transcriptions sont proches, car il existe un désir des contributeurs de bien écrire le soninké en suivant les conventions officielles. Néanmoins, on trouve des divergences dans les habituels points problématiques dont nous reparlons en détail dans la seconde partie de cet article (notation de la nasale palatale $n$ et de la nasale vélaire $\eta$, formes en $f$ et en $h$, alternances initiales, marques du pluriel, etc.). Par exemple, la consonne nasale vélaire [y] est transcrite, selon les textes, avec le symbole $\eta$ de l'Alphabet phonétique international (API), avec le Éta grec $\eta$, avec le digraphe $n w$ ou même avec le digraphe $n K$.

Le choix initial du site Soninkara.com de ne pas réviser les transcriptions des textes proposés a parfois abouti à certaines erreurs. C'est ainsi que, dans le glossaire soninké qui est présenté, la consonne nasale vélaire se retrouve confondue à d'autres lettres ( $n$ en position interne, $\tilde{n}$ en position initiale).

Exemples : bundanne au lieu de bundanye 'arc'; gajanne au lieu de gajanye 'combat';

ñame au lieu de yaame 'inondation'; etc. 
Cependant, en 2011, le Webmaster Fodyé Cissé estimait que l'ensemble de la transcription devrait être normalisée, dans le futur, selon les recommandations du Séminaire de Bakel de 1995.

\section{Les autres sites communautaires ou associatifs}

L'organisation des douze autres sites soninké ayant des rubriques culturelles et linguistiques est proche de celle de Soninkara.com. La plupart des sites et des blogs sont en français (ou quelquefois en anglais) avec une partie réservée à la langue soninké ; les sites ou blogs majoritairement ou exclusivement en soninké sont encore peu nombreux.

\section{Le site Soninkara.org}

[http://www.soninkara.org]

Comme mentionné précédemment, il a été créé en 2006, à partir d'une scission de Soninkara.com. Il est majoritairement en français, mais il est possible d'y télécharger un cours de soninké et l'on y trouve différentes sortes de textes dans cette langue, contes, proverbes et poèmes, par exemple un "Hymne à Dingha, ancêtre des Soninkés». De plus, sur le forum, beaucoup de débats sont en soninké. Les transcriptions utilisées, sans être homogènes, sont proches des conventions officielles.

Certaines rubriques sont identiques à celles de Soninkara.com. Par exemple, le glossaire soninké-français et le rapport du Séminaire de Bakel de 1995 sur l'harmonisation du soninké.

\section{Le site Soninkara.net}

[http://www.soninkara.net]

29 Le nom "Soninkara » est décidément très populaire. Ce site émane de l'association Sooninkara Renmun Kaho 'Union des enfants du pays soninké' de Kaédi en Mauritanie, ville dont sont originaires la plupart des linguistes soninké. Cette association (de même que son site) se consacre aussi bien à la promotion de la culture soninké qu'à des actions dans le domaine de la santé et du développement. La plupart des textes présentés sont en soninké ; ils respectent fidèlement l'alphabet officiel de la Mauritanie et du Sénégal, y compris pour la nasale palatale (transcrite $\tilde{n}$ ) et la nasale vélaire (transcrite $\eta$ ).

\section{Le site Soninkaxu}

[http://www.soninkaxu.com]

Il s'agit là d'un site basé à Paris, animé par Mahamadou Wackané Sacko, originaire de Nioro au Mali. Ce site se consacre uniquement à des questions culturelles : histoire, langue, traditions, origine des clans et familles, etc. Il est principalement en français, cependant on y trouve des textes bilingues soninké-français, notamment des contes. L'orthographe utilisée respecte les conventions officielles, sauf pour la nasale palatale et la nasale vélaire transcrites respectivement ny et $n w$. Ce site est lié au groupe Facebook Soninkaxu, très actif, ce qui lui permet d'élargir son audience auprès des internautes soninké. 


\section{Le site Asawan}

[http://www.asawan.org]

Asawan Kitaabinkonpe - Asawan Library - Bibliothèque Asawan

Il s'agit là du site de la SIL ${ }^{5}$, qui diffuse des textes en soninké (recueils de légendes, contes et proverbes, manuels de santé, traduction de textes bibliques, dictionnaire soninké/français/anglais). La transcription suit fidèlement le système officiel du Mali, notamment l'utilisation des caractères phonétiques pour la nasale palatale $(n)$ et la nasale vélaire $(\eta)$. Le site possède trois versions : en français, en anglais et en soninké. Notons aussi que le syllabaire soninké présenté se décline selon trois variantes: en «soninké standard » et en «soninké de l'Ouest» (avec des textes presque identiques), ainsi qu'en "soninké de l'Est ». Dans cette dernière version (élaborée pour la zone de Nioro à Nara), les $x$ initiaux sont remplacés par $q$, et les $f$ par $h$.

Même s'il est difficile d'être d'accord avec la tentative de proposer une graphie autonome pour le soninké de l'Est, il faut reconnaître que la SIL accomplit un véritable travail de recherche en n'hésitant pas à s'écarter des sentiers battus.

\section{Le site Soobe}

[http://www.soobe.8m.net]

Soobe est le site Web de l'«Association culturelle des Soninkés en Égypte » (ACSE) ou Sooninkon Maarinden Kafo Ejipt (SMKE) en soninké, qui avait eu un rôle pionnier dans le développement du soninké durant les années 1970. Le site Soobe est entièrement en soninké ; mais une grande partie des pages sont traduites en français et une petite partie en anglais. Par contre, la langue arabe n'est pas présente. L'organisation générale du site est proche de celle de Soninkara.com.

La transcription n'est pas unifiée, car des influences diverses viennent se rencontrer ici. La consonne nasale vélaire s'écrit tantôt $n w$ (selon l'usage pratiqué par l'ACSE durant les années 1970 et 1980), tantôt $\eta$; mais on trouve aussi un carré blanc ou un point d'interrogation (à cause de problèmes de transcodage).

\section{Le site Soninko.info}

[http://www.soninko.info]

Ce site, lié au village de Toulel (Mauritanie), est très intéressant. Il contient de nombreux articles en français, et aussi des contes et des poèmes en soninké (souvent écrits par le jeune écrivain malien Aliou Sy Sawané), ainsi que trois livrets en soninké qu'il est possible de télécharger et d'imprimer. Les textes en soninké sont très bien écrits en suivant le système officiel du Sénégal et de la Mauritanie, avec néanmoins certains problèmes pour la nasale palatale et la nasale vélaire (parfois remplacées par des carrés blancs).

\section{Le site Kouloun}

[http://kouloun.hautetfort.com]

C'est le site d'un programme de coopération décentralisée entre un lycée professionnel de Roubaix et les villages de Gabou, Diamou et Kouloun au Mali. Ce site essaye explicitement de développer la langue et la culture locale, ce qui est exceptionnel pour 
une ONG de ce type. Sur le site figure «Le Livre de Gabou » en soninké, très bien écrit ; mais on peut noter que la nasale palatale est transcrite avec la lettre sénégalaise $\tilde{n}$ et non pas avec la lettre malienne $n$ (qui est difficile à reproduire de façon générale).

\section{Le site Wagadu Saane}

[http://wagadusaane.4t.com]

Ce site, qui existe toujours, présente exclusivement les Journées culturelles soninké de Sélibaby (Mauritanie) qui s'étaient tenues du 13 au 15 mai 2004 (Wagadu Saane 'Étoile du Wagadou'). D'après le programme, il y eut de nombreux spectacles, prestations et allocutions en soninké. Mais tout le site est écrit en français, sauf un Hommage au linguiste disparu, Ousmane Moussa Diagana dit Dembo, qui est un poème en soninké.

\section{Le site Madi-Kaama}

[http://madikaama.org]

38 C'est le site de l'association Madi-Kaama-Musundo qui existe depuis 1997 et se réclame du philosophe soninké Madi-Kaama Kanouté (1827-1897). L'association est dirigée par Demba Traoré qui anime aussi la Radio rurale de Kayes.

L'association mène une importante action sociale dans la région de Kayes et les zones soninké voisines du Sénégal et de la Mauritanie. Elle organise des concours de nouvelles en soninké, et elle édite et vend des livrets en soninké. Sur le site, existe une rubrique où sont reproduits les textes primés lors des différents concours. Ces textes sont très bien transcrits selon les normes officielles du Mali; mais on peut supposer que le travail fut difficile, car on constate que la nasale palatale et la nasale vélaire ont été rajoutées de façon individuelle, avec une police différente.

\section{Le site Guidimakha Danka}

[http://guidimakhadanka.com]

C'est le site de l'association Guidimakha Danka 'Honneur du Guidimakha' dont le but est l'entraide entre les ressortissants du Guidimakha malien. Il n'y a rien sur la langue soninké, à part l'alphabet. Néanmoins, il est indiqué que l'association a aussi pour but de « contribuer activement à toutes les activités d'alphabétisation en langue locale ».

\section{Soninkara Kafo}

[http://soninkara-kafo.com]

41 Il s'agit là du site de l'« Association des Soninkés du Canada ", qui est essentiellement en français et un peu en anglais (notamment pour ce qui concerne la Gambie). On y trouve beaucoup d'informations politiques. Par ailleurs, l'association gère une radio émettant depuis Montréal.

Il existe une rubrique "Apprendre le soninké » où est reproduit le dictionnaire de la SIL, soninké-français-anglais. Figure aussi un « cours audio de soninké ».

\section{Wagadu Jikke}

[http://www.wagadu-jikke.org] 
43 l'association Wagadu Jikke 'Espoir du Wagadou', fondée en 2003 par des Soninkés de Kaédi et de Djéol (Mauritanie). Les objectifs étaient l'intégration et l'insertion de ses membres, et la promotion de la langue et de la culture soninké. Néanmoins, on constate l'absence d'une section « langue soninké ». Les quelques éléments culturels concernent plutôt la religion musulmane et l'histoire du Wagadou.

Nous avons souhaité mentionner ce dernier site car, même si la langue soninké n'est pas vraiment présente, il existe un désir déclaré d'en faire la promotion.

\section{Les blogs de militants de la langue et de la culture soninké}

Ces blogs sont essentiellement l'œuvre de jeunes Mauritaniens (peut-être car c'est en Mauritanie que la culture soninké se sent la plus menacée, face à la culture arabe).

- <http://diadiesoumare.over-blog.com>. Blog du jeune Diadié Soumaré de Agonitt en Mauritanie. Il contient des proverbes en soninké et une bonne analyse des problèmes de transcription (qui se réflètent dans ce blog où la lettre $y$ est souvent remplacée par un point d'interrogation).

- <http://sumaare.over-blog.com>. Blog de Demba Soumaré, entièrement en soninké, bien écrit selon la graphie de Mauritanie et du Sénégal. On a là l'exemple d'un blog fait par un artisan du bâtiment, ayant seulement fait des études primaires en français : il est devenu un militant culturel complètement investi dans la promotion de sa langue maternelle et est très actif sur tous les réseaux sociaux.

- <http://soonipoesie.musicblog.fr>. Blog de poésies soninké animé par Elhadji Ndiaye, l'un des principaux animateurs de l'Association pour la promotion de la langue et de la culture soninké (APS) dont le siège est à Saint-Denis, dans la région parisienne. À part quelques éléments de présentation, les textes sont entièrement en soninké.

- <http://guemou.blogvie.com>. Blog de Demba Camara consacré à Guémou, son village d'origine en Mauritanie. On y trouve des poèmes en soninké très bien transcrits selon le système officiel du Sénégal et de Mauritanie.

-<http://soumarezakariademba.blogspot.com>. Blog de Zakaria Demba Soumaré, originaire du village de Toulel (cité précédemment). Ce blog très fourni se consacre à toutes les facettes de la culture soninké, notamment l'histoire et l'organisation sociale. Néanmoins, le site est entièrement en français et il y a peu de choses sur la langue soninké elle-même. Notons que le son [x] est noté $h$. Exemples : niahamalo 'hommes de caste', hitinda 'annonceur d'un décès'.

Il faut noter qu'il existe beaucoup de poésies en soninké dans ces différents sites ou blogs.

En ce qui concerne la transcription de la langue, on peut observer dans la plupart des sites ou blogs un désir de suivre les normes officielles pour la notation du soninké. Néanmoins, bien souvent, il ne s'agit pas de l'orthographe institutionnalisée par tel ou tel État, mais plutôt des propositions du Séminaire de Bakel de novembre 1995, auquel ont participé des linguistes soninké venant de différents pays africains.

En pratique, les problèmes ou divergences viennent surtout de la notation de la nasale palatale $(\tilde{n} / n)$ et de la nasale vélaire $(\eta)$, des formes dialectales en [f] ou en $[\mathrm{h}]$, des alternances initiales et des marques du pluriel. Par exemple, la consonne nasale palatale est souvent notée avec la lettre $n$ surmontée d'un croissant comme en tchèque (̌n) et elle peut aussi être remplacée par le digraphe ny. 
Voyons maintenant les tendances linguistiques qui apparaissent en étudiant les sites de façon globale.

\section{$2^{\mathrm{e}}$ partie. Rôle d'Internet dans la standardisation du soninké écrit}

Lors de cette recherche, il nous est apparu que l'examen des sites Internet soninké est un bon moyen de voir quelles solutions sont adoptées par les scripteurs pour résoudre les cas de problèmes graphiques : variations dialectales ${ }^{6}$, notation de certains sons, etc. Les tendances dégagées fournissent ainsi des solutions pour la standardisation de la langue écrite.

Rappelons d'abord ci-dessous les principaux problèmes graphiques du soninké.

\section{La notation des sons $\Omega(\mathrm{N}$ palatal) et $\eta(\mathrm{N}$ vélaire $)$}

Ces sons sont notés respectivement $\tilde{n}$ et $\eta$ dans les alphabets officiels du Sénégal et de Mauritanie, et $n$ et $y$ dans l'alphabet officiel du Mali. Mais, au lieu de ces caractères, de nombreuses personnes utilisent d'autres symboles, par exemple les digraphes ny et $n w$.

\section{La notation orthographique des variantes phonétiques}

51 Nous présentons dans le tableau ci-dessous les principales variantes phonétiques existant en soninké. Nos exemples sont transcrits avec l'orthographe utilisée lorsque l'on veut fidèlement noter tel ou tel dialecte, tout en respectant les règles morphologiques et l'alphabet officiel.

\begin{tabular}{|c|c|c|c|}
\hline Régions & $\begin{array}{l}\text { Gadyaga } \\
\text { Mali et Sénégal } \\
\text { « soninké Ouest » }\end{array}$ & $\begin{array}{l}\text { Guidimakha } \\
\text { Mali et Mauritanie } \\
\text { « soninké Ouest » }\end{array}$ & $\begin{array}{l}\text { Khanyaga } \\
\text { Mali } \\
\text { «soninké Est » }\end{array}$ \\
\hline $\begin{array}{l}\text { 'son père' } \\
\text { 'ton père' } \\
\text { 'beau' }\end{array}$ & $\begin{array}{l}\text { a faaba } \\
\text { an paaba } \\
\text { faranpare }\end{array}$ & $\begin{array}{l}\text { a faaba } \\
\text { an faaba } \\
\text { faranfare }\end{array}$ & $\begin{array}{l}\text { a haaba } \\
\text { an paaba } \\
\text { haranpare }\end{array}$ \\
\hline $\begin{array}{l}\text { 'son cheval' } \\
\text { 'ton cheval' }\end{array}$ & $\begin{array}{l}\text { asi } \\
\text { an ci }\end{array}$ & $\begin{array}{l}\text { asi } \\
\text { an si }\end{array}$ & $\begin{array}{l}\text { a si } \\
\text { an } c i\end{array}$ \\
\hline $\begin{array}{l}\text { 'sa part', 'le sien' } \\
\text { 'ta part', 'le tien' } \\
\text { 'il est resté' } \\
\text { 'il reste' } \\
\text { 'descendre' }\end{array}$ & $\begin{array}{l}\text { a xalle } \\
\text { an qalle } \\
\text { a toxo } \\
\text { a wa toqqo } \\
\text { yanqa }\end{array}$ & $\begin{array}{l}\text { a xalle } \\
\text { an xalle } \\
\text { a toxo } \\
\text { a wa toqqo } \\
\text { yanxa }\end{array}$ & $\begin{array}{l}\text { a qalle } \\
\text { an qalle } \\
\text { a toxo } \\
\text { a wa toqqo } \\
\text { yanqa }\end{array}$ \\
\hline
\end{tabular}


NB : On a l'habitude de distinguer deux grandes variétés du soninké selon la présence ou non du son [f]. En soninké de l'Ouest, le son [f] est présent ; en soninké de l'Est, celuici est remplacé par [h] (indépendamment des transformations possibles en [p]).

Les sons $[\mathrm{x}]$ et $[\mathrm{q}]$ sont deux réalisation phonétiques d'un même phonème défini comme /oral, uvulaire, sourd/. En position initiale et en position postnasale, les réalisations sont variables selon les dialectes. Par contre, en position intervocalique, la réalisation est très stable : fricative si la consonne est simple, occlusive si la consonne est géminée. Théoriquement ces deux sons (réalisations d'une seule forme sous-jacente) pourraient être orthographiés avec une seule lettre ( $x$ ou q); néanmoins, l'habitude s'est prise de les distinguer.

\section{Les morphèmes du pluriel}

53 Nous présentons dans le tableau ci-dessous les trois systèmes de pluriels en présence $(u / o / n i, u / n u$ et $u / o / n u)$. Ceux-ci sont respectivement utilisés dans trois zones soninké prises en exemple : le Gadyaga (soninké de l'Ouest), le Khanyaga (soninké de l'Est) et la ville de Kaédi (soninké de l'Est influencé par le soninké de l'Ouest). En outre, certains locuteurs du soninké de l'Ouest utilisent le système $u / o / n u$.

\begin{tabular}{|l|l|l|l|}
\hline \multirow{2}{*}{ Régions } & $\begin{array}{l}\text { Gadyaga } \\
\text { Mali et Sénégal } \\
\text { «soninké Ouest » } \\
u-o-n i\end{array}$ & $\begin{array}{l}\text { Khanyaga } \\
\text { Mali soninké Est » } \\
u-n u\end{array}$ & $\begin{array}{l}\text { Maédi } \\
u-o-n u\end{array}$ \\
\hline 'un arbre' & yitte & yitte & yitte \\
'des arbres' & yittu & yittu & yittu \\
\hline 'un mouton' & jaxe & jaxe & jaxe \\
'des moutons' & jaxo & jaxu & jaxo \\
\hline 'un marabout' & moodi & moodi & moodi \\
'des marabouts' & moodini & moodinu & moodinu \\
\hline
\end{tabular}

NB : La communauté soninké de la ville de Kaédi (Mauritanie) utilise une variété de soninké de l'Est (avec le son [h]) alors qu'elle est située à l'ouest du pays soninké (dans une région peule). Elle avait connu une immigration de personnes pratiquant le soninké de l'Est, originaires notamment de la région de Nioro. Par ailleurs, les Soninkés originaires de Kaédi ont toujours été très actifs pour la promotion de leur langue.

\section{Quelques choix graphiques déjà observés}

Pour résoudre certains des problèmes cités ci-dessus, il existe déjà une pratique commune aux scripteurs soninké des différents pays. Par exemple, il y a un certain consensus pour ne pas noter les variations phonétiques initiales et pour privilégier en l'occurrence les formes du Guidimakha (même si elles sont minoritaires numériquement). De même les séquences phonétiques $[\mathrm{mb}]$ sont toujours notées $n b$. On constate aussi qu'il y a une tendance à noter la consonne géminée [qq] en position 
intervocalique au moyen de la consonne simple $q$, dans la mesure où l'opposition simple-géminée est aussi une opposition fricative-occlusive qui semble suffisante. L'opposition accompli/inaccompli [toxo/toqqo] pourra donc être notée toxo/toqo.

\section{Tendances décelées dans la transcription du soninké sur Internet}

Si l'on essaye de faire une synthèse globale de la façon dont le soninké est écrit sur Internet, on peut faire les constatations suivantes.

\section{Le pluriel des polysyllabes}

Dans les pluriels des polysyllabes, on trouve en majorité à la fois les morphèmes $u$ et $o$, comme en soninké de l'Ouest, alors que dans le soninké de l'Est, il existe uniquement des pluriels en $u$. Exemple : on trouve dans tous les cas la forme yittu 'arbres' qui est commune aux deux dialectes ; mais on trouve majoritairement la forme jaxo 'moutons', caractéristique de l'Ouest, et beaucoup plus rarement la forme jaxu, spécifique aux parlers de l'Est.

57 Une recherche globale sur Internet avec, successivement, la forme de l'Ouest soxaano, puis la forme de l'Est soxaanu (formes plurielles du mot soxaana 'cultivateur'), donne 14 occurrences pour soxaano et 2 occurrences pour soxaanu. De même, on trouve 104 occurrences de garanko et 4 occurrences de garanku (formes plurielles du mot garanke 'cordonnier'). Enfin, on trouve toujours la forme Sooninko 'les Soninkés' [73 occurrences sur le Web] et jamais la forme Sooninku [aucune occurrence].

\section{Le pluriel des monosyllabes et des mots d'emprunt}

58 Pour les pluriels des monosyllabes et des mots d'emprunt, selon les parlers, il existe soit le morphème -ni (uniquement à l'Ouest), soit le morphème -nu (à l'Est et dans certains parlers de l'Ouest). Or, sur Internet, on trouve les deux formes $-n i$ et $-n u$, avec une légère majorité pour -nu.

59 Si l'on fait une recherche globale sur Internet avec, successivement, la forme moodini puis la forme moodinu (formes plurielles de moodi 'marabout'), on trouve 19 occurrences pour moodini et 39 occurrences pour moodinu. La même recherche avec les formes noquni et noqunu (formes plurielles de noqu 'endroit') donne 5 occurrences de noquni et 16 occurrences de noqunu. Avec les formes banbarani et banbaranu 'les Bambaras', on ne trouve aucune occurrence avec -ni et 2 occurrences avec -nu.

Cela permet de conclure qu'Internet révèle une tendance à standardiser le pluriel soninké autour des trois morphèmes $-u,-o$ et $-n u$. C'est là un point qui n'avait pas été abordé dans le Séminaire de Bakel de 1995.

\section{Choix entre $f$ et $h$}

61 Quand il existe une correspondance entre des formes en [f] (Ouest) et des formes en [h] (Est), ce sont généralement les formes avec $f$ qui sont préférées, même si on trouve de temps en temps des formes avec $h$.

Exemples: falle 'dos, après' plutôt que halle; sefe 'parler' plutôt que sehe; fana 'premier' plutôt que hana ; faayi 'regarder, voilà' plutôt que haayi. 


\section{Les alternances phonétiques initiales}

Pour l'expression 'son père' :

a faaba : 18

a haaba: 2

Pour l'expression 'ton père' :

an faaba : 60

an paaba: 8

De même, pour le terme signifiant 'beau' :

faranfare : 152

faranpare : 13

haranpare : 1

\section{Choix entre $n s$ et $n c$}

La séquence phonologique /ns/ se réalise généralement [nc] et quelquefois [ns]. Or, on constate qu'elle est toujours transcrite ns sur le Web. C'est là un point non abordé dans le Séminaire de Bakel de 1995. Néanmoins cette transcription ns est exactement conforme à celle qu'on trouve dans les quatre lexiques ou dictionnaires de M. Bathily, O.M. Dantioko, O.M. Diagana et B. \& S. Smeltzer ; seul le lexique de Z. Dramé, G. Galtier \& O.M. Dantioko (1979) écrit $n c$.

Exemples: funse 'grain, graine'; tunsi 'accompagner'; dansa 'soutenir financièrement'; tansege 'quatre-vingts'.

\section{Choix entre $x$ et $q$}

Le phonème consonantique uvulaire sourd pose certains problèmes de transcription. Selon le contexte ou le parler, il peut se réaliser [x] ou [q]. Or l'on constate les tendances suivantes. En position initiale, il est toujours noté $x$, y compris par les locuteurs des parlers Est qui prononcent [q]. En position médiane simple, il est toujours noté $x$ (c'est un contexte où la prononciation [x] est générale). En cas de gémination (sa réalisation étant toujours occlusive), il est généralement noté $q$ (valant double consonne), quelquefois $q q$, et quelques rares fois $x x$. En position médiane prénasalisée, la séquence NC est généralement notée $n q$ et quelquefois $n x$; en revanche, lorsque le phonème consonantique uvulaire sourd est prénasalisé en position initiale (par la rencontre d'un autre mot possédant une nasale en position finale) et qu'il se réalise [q], la graphie $x$ est conservée.

De plus, on constate que l'ancienne transcription kh est très rarement utilisée. La transcription $x$ apparaît même dans des formes françaises. Ainsi, dans des textes en français, on trouve fréquemment la graphie Guidimaxa au lieu de la graphie 
administrative Guidimakha. On peut aussi rencontrer la lettre $x$ dans des textes soninké ayant une graphie francisante (exemple, xoulle au lieu de xulle 'blanc'). Tout cela indique que la graphie $x$ est devenue assez populaire et a dépassé le cercle étroit des linguistes. Le fait que la lettre $x$ est aussi utilisée en wolof, langue dominante au Sénégal, a pu être un facteur important.

Si l'on fait une recherche globale sur Internet avec, successivement, un certain nombre de formes optionnelles, l'on arrive aux résultats suivants.

Terme signifiant 'femme' (toujours prononcé [yaxare]) :

yaxare : 299

yakhare $: 129^{7}$

yahare: 4

Terme signifiant 'demain' ([xumbane] à l'Ouest, [qumbani] à l'Est) :

xunbane : 65

xunbani : 1

qunbane : 0

qunbani : 0

khunbane : 0

khunbani : 0

Terme signifiant 'vieillard' ou 'doyen' :

xirise : 60

qirise : 3

khirise : 0

Expression signifiant 'tes nouvelles?'

an xubaare : 4

an xibaare : 2

an qubaare $: 0$

an qibaare : 0

an khubaare : 0

an khibaare : 0

Expression signifiant 'sa langue' ([a xanne] à l'Ouest, [a qanne] à l'Est)

a xanne $: 8$

a qanne : 0

a khanne : 0

Expression signifiant 'ta langue' ([an xanne] dans le Guidimakha, [an qanne] partout ailleurs)

an xanne : 20

an qanne : 0

an khanne : 0

Terme signifiant 'faire descendre', 'héberger' :

yanqandi : 29

yanxandi : 8

yankhandi : 1

Terme signifiant 'dormir' :

xenqe $: 32$

xenxe: 5

khenkhe : 0

Terme signifiant 'laver' ([wanqi] ou [wanxi] à l'Ouest, [yanqi] à l'Est) :

wanqi : 32

wanxi » $: 2$

wankhi : 1

yanqi : 7

yanxi : 1

yankhi: 0 
Terme signifiant 'épouse' :

yaqe : 37

yaqqe : 19

yaxxe : 3

Terme signifiant 'endroit'

noqu : 105

noqqu : 13

noxxu : 4

Expression signifiant 'il reste' :

a wa toqo : 8

a wa toqqo : 1

a wa toxxo: 3

Expression signifiant 'il se couche' :

a wa saqa : 15

a wa saqqa : 2

a wa saxxa: 1

NB : Les tendances observées ici sont conformes aux recommandations du Séminaire de Bakel de 1995.

\section{La nasale vélaire $\eta$}

les alphabets officiels des trois pays, Mali, Sénégal et Mauritanie. Mais elle pose des difficultés d'impression. Aussi, elle est souvent notée $n w$, car la suite phonologique /$\mathrm{n}+\mathrm{w}-/$ se transforme automatiquement en [y] à la rencontre de deux mots et car le son [y] a la valeur phonologique d'une consonne double (prénasalisée) en position médiane (intervocalique). Il faut, du reste, remarquer que, lors de la réunion internationale de Bamako de mars 1966 sur les langues africaines, organisée par l'Unesco, on avait proposé de noter le son [y] du mandingue avec le digraphe $n w$.

69 À l'examen des sites Internet soninké, on se rend compte que le problème n'est pas encore résolu. On rencontre la lettre $y$ dans les sites dont les animateurs sont particulièrement compétents et vigilants. Mais on trouve assez souvent le digraphe $n w$. On trouve aussi la lettre grecque Éta ( $\eta$ ) et les notations $n g$ et $n g$. De plus, les webmasters n'ont pas toujours su gérer ce son et celui-ci est fréquemment remplacé par un carré blanc ou un point d'interrogation. Il arrive aussi que $\eta$ soit remplacé par la lettre $\tilde{n}$ ou par la simple lettre $n$ : cela est le cas dans le petit lexique soninké qui est reproduit à l'identique dans plusieurs sites, sans que des corrections aient été faites.

lon fait une recherche globale sur Internet avec, successivement, un certain nombre de formes optionnelles, l'on arrive aux résultats suivants (lorsque le son [y] est noté, car il faut tenir compte du fait qu'il est souvent remplacé par un carré blanc, un point d'interrogation ou autre chose).

Terme signifiant 'ami' :

menjanye : 15

menjanwe: 5

menjaye : 0

menjanne : 0

menjane : 0

menjanghe : 0

Terme signifiant 'hyène' : 
turunge: 5

turunwe: 7

turuye : 13

turunne : 2

turune: 9

turunghe: 1

Terme signifiant 'oiseau' :

yelinge: 2

yelinwe: 2

yeline: 7

yelinne : 2

yeline : 2

yelinghe : 0

\section{La nasale palatale $n$}

71 La consonne nasale palatale $\boldsymbol{n}$ existe dans tous les parlers soninké. Elle est notée $\tilde{n}$ dans les alphabets officiels du Sénégal et de Mauritanie, et $n$ dans l'alphabet officiel du Mali. Cependant, certains internautes la transcrivent ny (ce qui était la notation utilisée au Mali jusqu'à la fin des années 1980) car la lettre $n$ et même la lettre $\tilde{n}$ posent des difficultés d'impression. Notons que, lors du Séminaire de Bakel de 1995, il avait été proposé d'harmoniser la pratique du Mali avec celle du Sénégal et de la Mauritanie en utilisant uniquement la lettre $\tilde{n}$, mais cette recommandation n'a pas été entérinée par le Mali.

72 À l'examen des sites Internet soninké, on se rend compte que le problème n'est pas encore complètement résolu. Cependant, la notation $\tilde{n}$ arrive en tête. On trouve assez souvent le digraphe ny, et parfois les digraphes ni et $g n$. La notation $n$, officielle au Mali, est très peu utilisée par les scripteurs maliens qui préfèrent parfois utiliser la lettre $\tilde{n}$ par souci d'harmonisation avec leurs collègues du Sénégal et de Mauritanie (les occurrences de la lettre $n$ proviennent généralement des textes mis en ligne par la SIL de Kayes, qui est très bien outillée). Il arrive enfin que l'on utilise la lettre $n$ surmontée d'une demi-boucle ( ̌n comme en tchèque) pour remplacer $\tilde{n}$.

Si l'on fait une recherche globale sur Internet avec, successivement, un certain nombre de formes optionnelles, l'on arrive aux résultats suivants.

Terme signifiant 'artisan' ou 'homme de caste' :

ñaxamala: 97

nyaxamala: 30

jaxamala : 4

niaxamala: 34

gnaxamala: 3

Terme signifiant 'chameau' ou 'dromadaire' :

ñogome : 13

nyogome: 7

nogome: 5

niogome : 0

gnogome : 5

Terme signifiant 'se réjouir' :

ñaxali : 8

nyaxali : 5

jaxali : 6 
niaxali : 0

gnaxali : 0

\section{L'apport d'Internet pour la standardisation du soninké}

74 L'apport d'Internet, par rapport aux précédents acquis, se concentre sur quelques points. D'abord, quelques éléments grammaticaux tels que le pluriel pour lequel est privilégié le système $u / o / n u$. Puis, la préférence donnée au soninké de l'Ouest pour certains choix lexicaux en cas de divergence avec les parlers de l'Est. Ce soninké de l'Ouest est celui parlé au Gadyaga et au Guidimakha dans la « zone des trois frontières » (Mali, Mauritanie et Sénégal) qui est le principal foyer de l'émigration soninké. Il est vrai que la majorité des utilisateurs du Web semblent être des locuteurs du soninké de l'Ouest ou du soninké de Kaédi (qu'ils soient dans leur pays d'origine ou à l'étranger), mais non des locuteurs du soninké de l'Est. Le Web vient ainsi renforcer le fait que le soninké de l'Ouest était déjà privilégié auparavant dans d'autres secteurs tels que les émissions radiophoniques; en effet, il est parfois considéré comme le plus «pur » et le moins influencé par d'autres langues; de plus, il est commun à tous les pays soninkophones.

L'examen des sites Web montre aussi que beaucoup d'internautes, malgré l'existence du système Unicode, ont de la peine à reproduire les lettres phonétiques $y$ et $n$. Il est probable que l'utilisation croissante d'Internet par les Soninkés amènera une solution à ce problème, dans un contexte où les États perdent de leur influence : soit adoption par les utilisateurs d'équivalents tels que des digraphes; soit fabrication par les sociétés informatiques de nouveaux claviers mieux adaptés aux langues africaines, ainsi que beaucoup l'espèrent.

\section{Conclusion}

Un élément très frappant est l'inexistence des sites officiels gouvernementaux. On aurait pu s'attendre à ce qu'il y ait une présence de la langue soninké sur les sites officiels de pays tels que le Mali et le Sénégal qui ont encouragé l'introduction des langues nationales (dont le soninké) dans certains secteurs éducatifs (notamment l'enseignement primaire au Mali). Or, à part l'organigramme des ministères concernés, rien n'apparait lorsque l'on fait des recherches sur Internet. Le développement de la langue et de la culture soninké sur le Web est donc le fait de la société civile, d'associations et de personnes militantes, mais nullement le fait des autorités nationales.

Comme auparavant, l'expression écrite du soninké continue à être largement favorisée par le phénomène migratoire. Grâce aux migrants, l'usage de cette langue est apparu sur Internet, ce qui profite aussi à toutes les communautés soninké des pays d'origine. Lire et écrire le soninké est désormais possible pour tous les utilisateurs des nouveaux médias, sans intermédiaire institutionnel et malgré l'absence d'un secteur éditorial.

En examinant les sites Web de la communauté, on constate un processus de standardisation fondé sur la pratique et non la théorie. Internet vient ainsi confirmer ou compléter les apports des séminaires internationaux tels que celui de Bakel en 1995, ainsi que les travaux fait par des linguistes ou des pédagogues travaillant aussi bien dans les pays d'origine qu'à l'étranger. Cette standardisation s'opère largement autour 
du «soninké de l'Ouest » parlé au Gadyaga et au Guidimakha, dans la «zone des trois frontières » qui est le principal foyer de l'émigration soninké. En outre, ce soninké de l'Ouest est commun au Mali, à la Mauritanie et au Sénégal.

Aussi, malgré le partage des Soninkés entre différents États africains, malgré leur dispersion dans le monde, la langue continue à les unifier. Internet vient renforcer ce phénomène et est en train de devenir l'un des nouveaux véhicules de la culture soninké moderne.

\section{BIBLIOGRAPHIE}

BATHILY Mody, 2008, Dictionnaire soninké/français - français/soninké, Rouen, Librairie Dades, 460 p.

Collectif, 2003, Rapport du "Séminaire sous-régional sur l'harmonisation de l'orthographe du soninké », Bakel, Centre d'échanges et de formation pratique (CEFP), novembre 1995, 9 p.

DАNTIOKо Oudiary Makan, Dictionnaire soninké-français, Bamako, Éditions Jamana, 244 p.

DIAGANA Ousmane Moussa, 2011, Dictionnaire soninké-français, Paris, Karthala, 260 p.

DRAMÉ Z., GALTIER G., DANTIOKO O.M., Lexique soninké-français, Bamako, DNAFLA, 1979, 116 p.

GALTIER Gérard, Le Soninké (Sarakolé) : syllabaire et grammaire, Paris : chez l'auteur, 1971, 44 p.

GALTIER Gérard, Problèmes actuels de la transcription du soninké au Mali et au Sénégal, Niamey, Communication à la Réunion d'experts de l'Unesco sur la transcription des langues africaines, $1978,10 \mathrm{p}$.

GALTIER Gérard, 1980, Problèmes dialectologiques et phonographématiques des parlers mandingues, Thèse pour le doctorat de $3^{\mathrm{e}}$ cycle de linguistique, Université de Paris VII, $450 \mathrm{p}$.

GOMEZ MONT Carmen, 2007, Technologies de la communication et traditions sociales dans les communautés indigènes du Mexique, Hermès 48, pp. 213-219.

KIYINDOU Alain, 2011, « Réseaux socionumériques et solidarité ». Hermès 59, pp. 117-122.

KOULAYAN Nicole, 2006, « Les langues diasporiques et Internet : entre nouvelle territorialité, résistance identitaire et partage des savoirs ", Hermès 45, pp. 139-145.

PROULX Serge, 2008, "Des nomades connectés : vivre ensemble à distance », Hermès, 51, pp. $155-160$.

SMELTZER Brad, SMELTZER Susan, 2001, Lexique soninké-français-anglais - Index français-soninké et anglais-soninké, Bamako, SIL, $234 \mathrm{p}$.

VANNINI Laurent, LE CROSNIER, Hervé (dir.), 2012, Net.lang : réussir le cyberespace multilingue, Caen, C\&F Éditions et Réseau Maaya, 480 p. 


\section{NOTES}

1. À titre de comparaison, à la date du 20 septembre 2012, parmi les groupes communautaires de plus de 10 membres, nous avons dénombré environ 120 groupes peuls (y compris du Nigeria) et 15 groupes manjak (ethnie originaire de Guinée Bissau, ayant une diaspora très importante au Sénégal et en France).

2. En mars 2013, les effectifs de ces sept groupes étaient passés aux chiffres suivants : groupe "Mouvement Soninkara Wagadou Rèmou », 4250 membres; groupe «Marenmou Soninkho o Yanibaane Yade», 3639 membres; groupe "Soninkara Gnime: La Racine Soninké », 2292 membres; groupe «Sooninkara Reemou (liberté d'expression)», 1975 membres; groupe "Soninkara.com», 1476 membres; groupe «Rap Soninké», 1340 membres; groupe «Soninkaxu », 2126 membres. On peut remarquer l'augmentation très rapide des effectifs de ce dernier groupe en l'espace de quelques mois : la raison semble être son contenu de haute qualité, avec de nombreuses informations historiques et culturelles, extraites d'ouvrages de chercheurs et universitaires, spécialistes de l'Afrique. Par ailleurs, en mars 2013, un autre groupe Facebook avait dépassé les 1000 membres : « soninko nkeffo.com » avec 1333 membres.

3. En mars 2013, les effectifs de ces groupes étaient passés aux chiffres suivants : «Apprendre le Soninké de base », 971 membres ; « Académie Soninké », 249 membres ; « Soninkara : Proverbes / Citations Soninké », 221 membres ; "Sooninke Renme an Xannen Safa », 132 membres. De plus, deux nouveaux groupes, consacrés à l'étude de la langue soninké, commençaient à bien se développer »: "Apprendre le soninké » avec 257 membres; «O nan sefe sooninkanxannen kanma (parlons de la langue soninké) » avec 90 membres.

4. Il s'agit du site d'information «www.seneweb.com » qui fut créé en 1999 dans le but de relier tous les Sénégalais vivant soit au pays, soit à l'étranger. Il utilise exclusivement la langue française. Il demeure aujourd'hui l'un des sites africains les plus importants, par son audience.

5. "Société internationale de linguistique » en français ou "Summer Institute of Lingusitics » en anglais : société protestante dont l'objectif est de traduire la Bible dans toutes les langues du monde.

6. Les divergences dialectales apparaissent essentiellement au niveau phonétique superficiel. $\mathrm{Au}$ niveau phonologique sous-jacent du diasystème soninké, on retrouve généralement des formes unifiées. Néanmoins, on a généralement basé les diverses codifications graphiques du soninké sur le niveau phonétique et non sur le niveau phonologique, d'où par exemple la distinction entre la lettre $x$ et la lettre $q$ qui est purement phonétique et non phonologique.

7. Le résultat 129 pour yakhare n'est pas vraiment significatif, car il s'agit essentiellement de sites en français qui signalent quelques mots soninké en utilisant une orthographe française.

\section{RÉSUMÉS}

Depuis quelques années la langue soninké a fait son apparition sur Internet. On y trouve des sites d'associations et des blogs de militants qui se consacrent à la défense et à l'illustration de leur langue et de leur culture. Le principal de ces sites est Soninkara.com (basé à Paris) qui a été créé en 2000. Parmi les autres sites, il faut notamment citer Soobe, le site de l'Association culturelle des Soninkés en Egypte. Ces sites ont souvent été créés pour des besoins de contact dans une diaspora dispersée aux quatre coins du monde. Ils donnent une tribune à la langue soninké, qui avait eu de la peine, jusqu'à présent, à trouver sa place dans l'édition papier. Ils permettent aussi 
une standardisation fondée sur la pratique et non la théorie. Celle-ci s'opère autour du «soninké de l'Ouest» parlé au Gadyaga et au Guidimakha dans la «zone des trois frontières » (Mali, Mauritanie et Sénégal) qui est le principal foyer de l'émigration soninké.

Since a couple of years, the Soninke language has appeared on the Internet. One can find sites of associations as well as blogs of activists devoting themselves to the promotion of their language and culture. The most important among the sites is Soninkara.com (based in Paris), created in 2000. Among others, Soobe, the site of the Soninke Cultural Association in Egypt, is to be specially mentioned. These sites were often created for the need of communication inside the Soninke diaspora scattered all over the world. They provide a written forum to the Soninke language which had, until recently, great difficulties in acquiring printed organs. They also bring forth a standardization based on the practice, rather than on theory. This emerging standard version is based on the "Western Soninke" spoken in Gajaga and Gidimakha, in the "three boundaries" area (Mali, Mauritania and Senegal) which represents the main core of Soninke emigration.

УЖЕ В ТЕЧЕНИЕ НЕСКОЛЬКИХ ЛЕТ ЯЗЫК СОНИНКЕ ПРИСУТСТВУЕТ В ИНТЕРНЕТЕ. ИМЕЮТСЯ САЙТЫ РАЗЛИЧНЫХ АССОЦИАЦИЙ, БЛОГИ АКТИВИСТОВ, ПОСВЯЩЁННЫЕ ЗАЩИТЕ И ПРОПАГАНДЕ ЯЗЫКА И КУЛЬТУРЫ СОНИНКЕ. ГЛАВНЫМ САЙТОМ МОЖНО СЧИТАТЬ Soninkara.com (БАЗИРУЮЩИЙСЯ В ПАРИЖЕ), СОЗДАННЫЙ В 2000 ГОДУ. СРЕДИ ДРУГИХ СЛЕДУЕТ ОСОБО УПОМЯНУТЬ САЙТ КУЛЬТУРНОЙ АССОЦИАЦИИ СОНИНКЕ ЕГИПТА, Soobe. ОБЫЧНО ТАКИЕ САЙТЫ СОЗДАЮТСЯ ДЛЯ НУЖД КОММУНИКАЦИИ ВНУТРИ ДИАСПОРЫ, РАССЕЯННОЙ ПО ВСЕМУ МИРУ. ОНИ ПРЕДОСТАВЛЯЮТ ТРИБУНУ ЯЗЫКУ СОНИНКЕ, КОТОРЫЙ ДО СИХ ПОР ТАК И НЕ ОБРЁЛ ПЕЧАТНЫХ ОРГАНОВ. ОНИ ТАКЖЕ ДЕЛАЮТ ВОЗМОЖНОЙ СТАНДАРТИЗАЦИЮ, ОСНОВАННУЮ НА ЯЗЫКОВОЙ ПРАКТИКЕ, А НЕ НА ТЕОРИИ. СТАНДАРТНЫЙ ВАРИАНТ ЯЗЫКА ВЫРАБАТЫВАЕТСЯ НА ОСНОВЕ «ЗАПАДНОГО СОНИНКЕ», НА КОТОРОМ ГОВОРЯТ В РАЙОНАХ ГАДЬЯГА И ГИДИМАХА В «ЗОНЕ ТРЁХ ГРАНИЦ» (МАЛИ, МАВРИТАНИИ И СЕНЕГАЛА), ОСНОВНОГО ОЧАГА СОНИНКСКОЙ ЭМИГРАЦИИ.

\section{INDEX}

Keywords : Soninke, Standardization, Diaspora, Emigration, Internet, Civil Society

Thèmes : soninké

motsclesru СОНИНКЕ, СТАНДАРТИЗАЦИЯ, ДИАСПОРА, ЭМИГРАЦИЯ, ИНТЕРНЕТ, ГРАЖДАНСКОЕ ОБЩЕСТВо

Mots-clés : standardisation, diaspora, Internet, émigration, société civile

\section{AUTEUR}

GÉRARD GALTIER

INALCO / USPC

gerardgaltier@noos.fr 